\title{
Research on Genetic Algorithm Solving Multi-modal Optimization \\ Problem
}

\section{Shoubai Xiao}

\author{
Jiangxi University of Technology, Nanchang 330098, China
}

\begin{abstract}
Keywords: Genetic algorithm; Multi-modal optimization problem; Fitness difference of local optima; Searching space scale; Chaos.
\end{abstract}

\begin{abstract}
It has become a widely concerned problem in genetic algorithm and even evolutionary computing field that how to apply genetic algorithm to solve multi-modal optimization problem, and it is the basis for genetic algorithm theory and practical application. Achievements in this aspect are emerging in endless, while theoretical researches are comparatively less. Especially there is no research on influences of external parameters to performances of applying genetic algorithm to solve multi-modal optimization problem yet. This article mainly carries out theoretical researches on this aspect and generalizes corresponding conclusions. The generalized theoretical results are used to improve searching performance of genetic algorithm in solving multi-modal optimization problem. Main research contents of this article include brief review of multi-modal optimization problem and genetic algorithm, analysis of research status of applying genetic algorithm to solve multi-model optimization problem, and introduction to basic theory and development trend of genetic algorithm. In this article, two kinds of complicated evolutionary systems based on infinite population model of genetic algorithm are analyzed, two kinds of new population evolutionary systems are built on this basis, and dynamic equation under the condition of single-gene is derived as well.
\end{abstract}

\section{Brief Introduction}

Growing from nothing and from weak to strong, evolutionary computing methods represented by genetic algorithm and their theoretical researches have gone through more than 40-year history. During this period, heuristic optimization algorithms such as simulated annealing algorithm, ant colony algorithm, immune algorithm and particle swarm optimization algorithm emerged one by one and they had even better effects in solving certain problems. However, by comparing statistics of researches in each period, it can be seen that no matter from the quantity of articles or research depth, evolutionary computing is always in the dominant position. If it is only a kind of method of optimizing numerical value and systematic design, it would not attract longstanding attentions from so many scholars. One reason for eternity of evolutionary algorithm researches is that evolutionary algorithm itself is a simulation of biological evolution and genetic process, and that researches on genetic algorithm can help better understandings on essence of biological evolution. This is why scholars are also interested in some detailed features of it. Questions like "is it better with more crossovers or more variations" are still argued today, and different opinions would derive different research branches, thus making evolutionary computing field prosperous. Long-term accumulations have set sound theoretical basis for follow-up studies of evolutionary computing, which is also an important reason for its continuous development. 
Multi-modal optimization problem exists in every aspect of the actual production and life, but just in recent 10 or 20 years, it actually became a subject to which attached importance. Development of decision science defines existence value of this problem. In the past, people only needed one of the global optimal solutions, but they increasingly realize that information grasped by system analyzers and decision makers are asymmetric, and system analyzers need to provide as many alternatives as possible for decision makers to make judgments according to grasped special information. Moreover, due to all kinds of objective limitations, some global optimal solutions are not applicable in practical application, so more global optimal solutions and local extremum solutions are needed as alternatives. A large number of non-classical algorithms are put forward, providing conditions for solving this problem. Former classic mathematical methods could only search single local extremum solution, which lacked global searching ability, therefore, heuristic search algorithms such as genetic algorithm made up this defect. All in all, along with development of operational research and system science and their applications in actual problems, multi-modal optimization problems are increasingly attracting people's attentions.

How to apply GA to solve multi-modal optimization problem has become a research focus in GA field and with very important practical significances. Achievements in this aspect are emerging in endless, while theoretical researches are comparatively less. Especially there is no research on influences of external parameters to performances of applying GA to solve multi-modal optimization problem yet. Therefore, strengthening theoretical research on this aspect is very important for further improving GA.

In this article, influences of attraction domain of local extremum solutions to GA convergence results and influences of parameter searching space scale to GA searching capability are discussed, and the conclusions speak volumes for necessity of reasonable division of solution space. In combination with orthogonal design method, GA based on reasonable division of solution space is put forward, and simulation experiments verify validity of GA in solving multi-modal optimization problem.

\section{Features of Genetic Algorithm}

Main features of GA are as follows:

(1) Loose limitations of GA to optimization objects. Because after selecting coding scheme, the one processed by GA is code-set of parameters other than function itself, so there are no specific requirements for nature of optimization function, which is different from requirements of traditional optimization methods for continuity and differentiability of optimization functions.

(2) Searching initial point of GA is initial population other than single initial point, which method is in favor of search process jumping out of local extreme point with large probability, thus improving ability of GA in searching global optimal solutions.

(3) GA possesses implicit parallelism. Considering solution process of GA from the modal aspect, at the same time when processing individuals of the colony, individual modes are process as well, showing feature of parallel processing.

(4) GA construction is simple and easy to realize, it has no excessive limits to specific operation environment, and it can be realized on different hardware and software platforms.

(5) In search process of GA, probability transformation criterion is adopted other than certainty criterion. This criterion is only a tool to lead the search process towards more optimal area of searching space. It seems a blindness search, but actually it has specific and clear search direction. 
(6) GA possesses typical dynamic features. Genetic operators will generate complicated dynamic behaviors during algorithm operational process, and GA will show up quasi periodicity and chaotic characteristics when solving some problems.

The above mentioned features make GA simple to use, strong in robustness, and easy for parallelization and theoretical analysis, thus can be widely applied in reality.

\section{Schemata Theorem of Genetic Algorithm}

Holland firstly put forward Schemata Theorem in 1975, and so far Schemata Theorem is greatly developed. Applications of Markov Model, general statistic models and analytic solution model enrich achievements of Schemata Theorem.

According to coding viewpoint of Holland, binary coding based on character set $\{0,1\}$ is the optimal coding scheme. In GA, practical problem is generally expressed as bit string space composed of character set $\{0,1\}$, namely $S^{1}=\{0,1\}^{l}$. Schema $\mathrm{H}$ is a set composed of bit strings in $S^{1}$ with common characteristics, and is an element of expanded bit string space added with wildcard character $* S_{e}{ }^{l}=\{0,1, *\}^{t}$. Introduction of schemata provides a useful tool for similarity measurement and theoretical analysis of bit strings with finite length defined based on finite character set. Through analyzing influences of genetic operators to survival quantity of schemas, Holland worked out famous Schemata Theorem.

Schemata Theorem: under functions of selection, crossover and variation of genetic operators, those schemas which with low order and short distance and exceed average fitness value will increase by exponential order along with increase of iterations.

$$
E(H, t+1)=N \cdot P\left(H_{s} t+1\right) \geq N \cdot P\left(H_{s} t\right)\left(1-P_{m}\right)^{o(H)} \cdot\left[1-P_{c} \frac{D(H)}{E(H)}\left(1-P\left(H_{s} t\right)\right)\right]
$$

Schemata Theorem can be expressed as the following formula:

$$
E(H, t+1)=N \cdot P\left(H_{s} t+1\right) \geq N \cdot P\left(H_{s} t\right)\left(1-P_{m}\right)^{o(H)} \cdot\left[1-P_{c} \frac{D(H)}{E(H)}\left(1-P\left(H_{s} t\right)\right)\right]
$$

Wherein, $\mathrm{E}(\mathrm{H}, \mathrm{t}+1)$ represents quantity of individuals which belong to Schemata $\mathrm{H}$ and contained in $\mathrm{t}+1$ generation population, $\mathrm{N}$ represents population size, $\mathrm{p}(\mathrm{H}, \mathrm{t})$ represents existence probability of Schemata $\mathrm{H}$ in $\mathrm{t}$ moment, $P_{m}, P_{c}$ respectively represent variation and crossover probabilities, $\mathrm{O}(\mathrm{H})$ and $\mathrm{D}(\mathrm{H})$ represent order and dimension of Schemata $\mathrm{H}$ respectively.

Even so, Schemata Theorem still can not explain specific mechanism from GA convergence to optimal solution, so Holland put forward famous Building Blocks Hypothesis in further research. Building blocks mean those schemas which with low order and short distance and exceed average fitness value.

Building Blocks Hypothesis: under functions of evolutionary operators, building blocks can form schemas with high order, long distance and fitness value higher than average through restructuring, thus constituting larger building blocks.

Schemata Theorem accurately describes competitive relation between schemas within GA population, while Building Blocks Hypothesis indicates inheritance and restructuring relations 
existing between effective genes. Based on analysis, Holland and Goldberg put forward that number of schemas with effective process is $O\left(n^{3}\right)$, and this nature is called as implicit parallelism. The above mentioned theory, called as Schemata Theorem, is a relatively systematic theory to explain GA optimizing principal.

\section{Influences of Attraction Domain Size of Local Extremum Solutions to Results of GA Convergence}

Generally speaking, there is always a valley bottom between peak values of two local extremum solutions, whose fitness value is smaller than the around values. Taking local extremum solution as center and adjacent valley bottoms as boarders, thus so-called attraction domain of this local extremum solution is formed. Fitness values from valley bottom to central peak are increasing. When solving multi-modal function optimization problem, if individuals of GA initial population are all in attraction domain of a certain local extremum solution, or if individual diversities of search population decrease along with increase of evolutionary algebra and the residual individuals are all in attraction domain of a certain local extremum solution, it is probably that GA would fall into local extremum solution. This probability is related to attraction domain size of local extremum solution, the larger attraction domain of local extremum solution is, the higher probability of algorithm falling into local extremum solution is. Generally, it is hard to tell the exact position of valley bottom, so attraction domain size is estimated by half distance between local extremum solution and its adjacent local extremum solution.

How will attraction domain size of each local extremum solution affect search results of GA? This question will be analyzed through the following specific examples.

Firstly, some typical multi-modal optimization functions are introduced as follows.

$$
\begin{array}{r}
\max f_{0}(x)=\sin ^{6}(\pi \sqrt{x}) x \in[Q] \\
x^{*} \in\{0,1,0,3,05,0,7,0,9\}, f_{0}\left(x^{*}\right)=1.0 。 \\
\max f_{0}(x)=\sin ^{6}(5 \pi x), x \in[0,1]
\end{array}
$$

Test function $f_{0}$ is a function defined within interval of $[0,1]$ and with multi-extremum solutions, distances between every two extremum solutions are equal, and function values of all extremum solutions are equal. $x^{*} \in\{0,1,0,3,05,0,7,0,9\}, f_{0}\left(x^{*}\right)=1$. 0 。

$$
\begin{aligned}
& \max f_{2}(x)=e^{-2 \times \ln 2 \times(x-0.1 / 0.8)^{2}} \times \sin ^{6}\left(5 \pi\left(x^{3 / 4}-0.05\right)\right), x \in[0,1] \\
& x^{*} \in\{0.0797,0.2467,0.4506,0.6814,0.9339\}, \\
& f_{2}\left(x^{*}\right) \in\{0.9991,0.9545,0.7662,0.4809,0.2217\} \\
& \max f_{2}(x)=e^{-2 \times \ln 2 \times(x-0.1 / 0.8)^{2}} \times \sin ^{6}\left(5 \pi\left(x^{3 / 4}-0.05\right)\right), x \in[0,1]
\end{aligned}
$$


Test function $f_{2}$ is a function defined within interval of $[0,1]$, distances between every two extremum solutions are unequal, and function values of all extremum solutions are unequal either. $x^{*} \in\{0.0797,0.2467,0.4506,0.6814,0.9339\}$, $f_{2}\left(x^{*}\right) \in\{0.9991,0.9545,0.7662,0.4809,0.2217\}$

$$
\begin{gathered}
\max f_{3}(x)=\sin ^{6}\left(5 \pi\left(x^{3 / 4}-0.05\right)\right) x \in[Q] \\
x^{*} \in\{0.0797,0.2467,0.4506,0.6814,0.9339\}, f_{0}\left(x^{*}\right)=1.0 。 \\
\max f_{3}(x)=\sin ^{6}\left(5 \pi\left(x^{3 / 4}-0.05\right)\right), x \in[0,1]
\end{gathered}
$$

Test function $f_{3}$ is a function defined within interval of $[0,1]$, distances between every two extremum solutions are unequal, while function values of all extremum solutions are equal. $x^{*} \in\{0.0797,0.2467,0.4506,0.6814,0.9339\}, f_{0}\left(x^{*}\right)=1.0$ 。

Function $f_{0}$ and $f_{3}$ have the same searching space and number of local extremum solutions, and in relative terms of each function, fitness values of local extremum solution are equal, so the only factor affecting search probability of each local extremum solution is attraction domain size. Attraction domain size is measured as half of the sum distances between certain local extremum solution and adjacent two local extremum solutions (half distance).

Observations show that each local extremum solution of test function $f_{0}$ is equidistant, so their attraction domain sizes are the same. 100 times of searches were carried out to functions via SGA, and times of each extremum solution showed up in search results were compared.

\section{Mathematical Description of Multi-modal Optimization Problem}

In order to research mechanism of applying GA to solve multi-modal optimization problem, model shall be built and analyzed, therefore it is necessary to describe concepts of multi-modal optimization problem with mathematical language.

In actual applications, GA mainly adopts the following coding schemes: binary coding, real number encoding, large character set encoding and assembly coding. In this article, binary coding is adopted. This is because various different coding forms only affect efficiency of evolutionary operators other than essence of solving mechanism of GA, besides, as a frequently-used major coding scheme, binary coding has incomparable advantages in theoretical analysis process. Under the condition of binary coding, multi-modal optimization problem can be described as follows.

$$
b \in I B^{l}=\{0,1\}^{l}, \text { 都有 } 0<\mathrm{f}(\mathrm{b})<+\infty, \text { 并且 } \mathrm{f}(\mathrm{b}) \neq \mathrm{cons} \text { 。 }
$$


Multi-modal optimization problem can be described as solving $\left\{b \mid f(b)\right.$ 为极大值, $\left.\mathrm{b} \in I B^{l}\right\}$. Wherein, $\mathrm{f}(\mathrm{b})$ is a multi-modal function, for all $b \in I B^{l}=\{0,1\}^{l}, 0<\mathrm{f}(\mathrm{b})<+\infty$ and $\mathrm{f}(\mathrm{b}) \neq$ cons.

During research process, it is very important to establish a theoretical basis for multi-modal optimization algorithm convergence. Different from concept of "Global Convergence" which can only converge one global optimal solution, convergence of multi-modal optimization algorithm shall be interpreted as: along with increase of evolutionary algebra, algorithm can finally get all extremum solutions in domain of definition with probability of " 1 ". Convergence which can converge all extremum solutions in domain of definition is called "Complete Convergence".

$$
\lim _{t \rightarrow \infty} \coprod_{i=1}^{m} P\left(\Phi_{i} \in Z(t)\right)=1
$$

With regard to multimodal function referred in problem description, given that there are $\mathrm{m}$ extremum solutions in domain of definition which are recorded as $\Phi_{1}, \Phi_{2} \ldots \Phi_{m}$, and the $\mathrm{t}$ generation population is written down as $\mathrm{Z}(\mathrm{t})$. When and only when the following condition is satisfied, algorithm is complete convergence:

$$
\lim _{t \rightarrow \infty} \coprod_{i=1}^{m} P\left(\Phi_{i} \in Z(t)\right)=1
$$

\section{Conclusions}

Evolutionary computing is a kind of heuristic random search optimization method derived from biological evolution and enlightenment of modern genetics. As a new interdisciplinary subject, evolutionary computing has wide application prospect, so further researches on its theoretical basis shall be strengthened. It is a problem to be solved that how to research theoretical basis of GA and to apply theoretical results into algorithm improvement. At present, How to apply GA to solve multi-modal optimization problem has become a research focus in GA field and with very important practical significances. Achievements in this aspect are emerging in endless, while theoretical researches are comparatively less. Especially there is no research on influences of external parameters to performances of applying GA to solve multi-modal optimization problem yet. This article mainly carries out theoretical researches on this aspect and applies theoretical results to improve searching performance of genetic algorithm in solving multi-modal optimization problem. Main research contents of this article are as follows:

(1) Brief review of multi-modal optimization problem and genetic algorithm, analysis of research status of applying genetic algorithm to solve multi-model optimization problem, and pointing out problems to be solved. Detailed introduction to basic theory and discussion on development trend of genetic algorithm are made as well.

(2) Influences of attraction domain of local extremum solution to GA convergence results and influences of parameter searching space to GA searching performance are discussed, and the conclusions speak volumes for necessity of reasonable division of solution space. Based on the above mentioned theoretical results and in combination with orthogonal design method, GA based on reasonable division of solution space is put forward. Firstly, orthogonal design is adopted to explore 
searching space so as to determine appropriate space division strategy, and to divide searching space based on this strategy; then single search to each subspace is carried out with GA respectively, and recursive call of GA to each subspace is made according to obtained results; lastly effects of GA to multi-modal optimization problem are verified through simulation experiments.

\section{Acknowledgement}

This article is funded by science and technology planning project in Jiangxi Province- Systematic Research of Intellectual Property Rights Trading Center of Jiangxi Province (No.: 20143BBM26128) and Jiangxi University of Technology [No. ZR13ZD03].

\section{References}

[1] Zhong Kailai (writer), Liu Wen (translator), Probability Theory Course, Shanghai, Shanghai Science and Technology Press, 1989

[2] Shi Renjie, Markov Chain Basis and Application, Xi'an, Xidian University Press, 1992

[3] Li Minqiang, Kou Jisong, Analysis on Fraudulence of Model of Genetic Algorithm, Science China No. 5, vol.32(1), 2002.95-102

[4] Yang Haijun, Li Minqiang, Modal Form Invariance and Accurate Schemata Theorem, Science China No. 5, Vol.33(8), 2003.707-714

[5] Bethke A D.Genetic Algorithms as Function Optimizers. Dissertation AbstractsInternational, 1981, 41(9), 3503(B).

[6] Poli R. Hyperschema Theory for GP with One-point Crossover, Building Blocks, and Some New Results in GA Theory. Euro GP 2000:2000， 163-180

[7] Li Minqiang, Kou Jisong, Synergy Multi-population Genetic Algorithm of Multi-modal Function Optimization, Acta Automatica Sinica, Vol.28(4), 002.497-504

[8] Zhang Wenxiu, Liang Yi (compiler), Mathematic Foundation of Genetic Algorithm, Xi'an, Xi'an Jiaotong University Press, 2000

[9] Liu Hu, Zhang Huanchun, Jing Yazhi, Zhu Lili, Parameters of Fuzzy Genetic Algorithm of Paralleled Multi-population, Jilin College Journal, Vol.23(6), 2005.629-634

[10] Wang Pin, Gu Peiliang, Liang Jiaojie, Qian Weiguo, Application of Simulated Annealing Modified GA in Investment Decision-making in Stock Market, Tianjin University Journal, vol.33(1), 2000.51-54 